in similar disease phenotypes ${ }^{1}$ and that the R120G $\alpha$ B-crystallin mutation results in aggregation of desmin filaments ${ }^{3}$. Most sHSPs ( $\alpha$ B-crystallin, HSP27, HSP22, HSP20, MKBP, HSPB3, cvHSP) are particularly abundant in muscles and heart, where they may comprise $3 \%$ or more of total protein. At least three sHSPs ( $\alpha \mathrm{B}$-crystallin, HSP27, HSP22) are abundant in different nerve cells, and $\alpha \mathrm{B}$-crystallin and HSP27 accumulate in individuals with various neurodegenerative disorders, including Alzheimer, Parkinson and Alexander diseases and multiple sclerosis. Thus, that mutations in sHSPs would cause neuromuscular disorders is not entirely unexpected. More baffling is the decade-long ignorance of sHSPs by neuromuscular researchers.

Although a number of interacting partners have been identified, the most obvious interactions involving sHSPs occur with each other, resulting in formation of homo- and hetero-oligomeric complexes. Indeed, formation of complexes with a wide range of molecular masses is the most pronounced, though least understood, characteristic of sHSPs. These complexes are dynamic structures, with subunits exchanging rapidly. This situation may contribute to the difficulty of understanding the cellular function of sHSPs. Whatever their precise role is at the molecular level, the properties of the ten sHSPs are probably not identical. For example, the sequences of the C-terminal tails of the ten proteins, which are distinguished by their high conformational flexibility, are very different and probably confer unique properties on each sHSP. The fact that mutations in different sHSPs are associated with similar, but not identical, diseases support this idea of distinct roles for these proteins. Moreover, the relatively late onset of some neuromuscular diseases, which suggests an accumulative tissue damage pattern, indicates a more indirect, perhaps protective role of the wild-type sHSPs rather than an immediate role in proper tissue function.

\section{What's next?}

What is the degree of functional redundancy and difference among the ten mammalian sHSPs? Can sHSPs other than HSP27, $\alpha \mathrm{B}-$ crystallin, $\alpha \mathrm{A}$-crystallin and HSP20 protect cells from stress? How does the composition of sHSP complexes vary in different cell types? Do complexes vary during development or in response to physiological signals? Is the composition of complexes regulated, thus allowing a fine-tuning of the functions of sHSP complexes? What is the functional importance of various hetero-oligomeric complexes? Indeed, the different expression patterns of sHSPs in different organs and tissues and the idea that probably not all sHSPs are stress-inducible suggest that the composition and functions of sHSP complexes may be cell- and stress-specific.

Regarding the heterogeneous group of motor neuropathies and muscular dystrophies of unknown cause, the identification of more sHSP mutations can be expected. Although poor understanding of sHSP functions will impede development of therapeutic strategies in the near future, there is reason to be optimistic that much will be learned about the molecular and cellular role of sHSPs in the coming decade, as the importance of this protein superfamily becomes more widely recognized.

1. Irobi, J. et al. Nat. Genet. 36, 597-601 (2004).

2. Evgrafov, O.V. et al. Nat. Genet. 36, 602-606 (2004).

3. Vicart, P. et al. Nat. Genet. 20, 92-95 (1998).

4. Selcen, D. \& Engel, A.G. Ann. Neurol. 54, 804-810 (2003).

5. Berry, V. et al. Am. J. Hum. Genet. 69, 1141-1145 (2001).

6. Litt, M. et al. Hum. Mol. Genet. 7, 471-474 (1998).

7. Pras, E. et al. Invest. Ophthalmol. Vis. Sci. 41, 3511-3515 (2000)

8. Mackay, D.S., Andley, U.P. \& Shiels, A. Eur. J. Hum Genet. 11, 784-793 (2003).

9. Mörner, C.T. Hoppe Seylers Z. Physiol. Chem. 18, 61-106 (1894).

10. Kappe, G. et al. Cell Stress Chaperones 8, 53-61 (2003).

11. Fontaine, J.M., Rest, J.S., Welsh, M.J. \& Benndorf, R. Cell Stress Chaperones 8, 62-69 (2003).

\title{
Sex and the genome
}

\author{
Valerie Reinke
}

The genetic composition of sex chromosomes has been deciphered, in part, through large-scale gene expression studies in different species. A new study in mice adds a missing piece of the puzzle: the composition of the $X$ chromosome in mammals is influenced by inactivation of the sex chromosomes during male meiosis.

Sex complicates many aspects of life but is sufficiently beneficial that virtually every eukaryotic species uses it. An especially sticky consequence is that an unequal complement of sex-determining chromosomes exists between the two sexes. One sex, typically female, carries two X chromosomes, and the other sex, usually male, has a single $\mathrm{X}$ chromosome (accompanied by a $\mathrm{Y}$ chromosome in some, but not all, species). This inequality puts the sex chromosomes in an evolutionarily vulnerable position.

Valerie Reinke is in the Department of Genetics, Yale University School of Medicine, New Haven, Connecticut, USA.

e-mail: valerie.reinke@yale.edu
Two hypotheses propose different outcomes for sex-linked genes that are preferentially expressed in one sex over the other. Rice's hypothesis ${ }^{1}$ states that, because males carry a single $\mathrm{X}$ chromosome, any recessive allele arising on the $\mathrm{X}$ that gives males a reproductive advantage is immediately available for positive selection. Conversely, any negative effects of that allele in females are masked by the presence of a second X chromosome. Thus, genes expressed preferentially in males are more likely to be present on the $\mathrm{X}$ chromosome. An alternate hypothesis states that the $\mathrm{X}$ should be feminized because the $\mathrm{X}$ chromosome is present in females 50\% more frequently than in males, providing evolution with more opportunity to act on genes benefiting females. Thus, genes with female-biased expression should reside on the $\mathrm{X}$ chromosome. So which hypothesis is correct, or are the two hypotheses mutually compatible? In this issue, Pavel Khil and colleagues ${ }^{2}$ look at sex-biased expression profiles in mice and show that, as for many relationships, the answer involves a compromise.

\section{On again, off again}

Khil et al. adopted a computational approach using publicly available mouse expressedsequence tag and microarray databases to define genes expressed preferentially in various somatic and gonadal tissues. They then 
mapped these genes onto the 20 mouse chromosomes and looked for biases in location. Genes expressed in various somatic tissues, such as liver and lung, showed no significant bias, but genes expressed preferentially in gonadal tissues told a different story. In particular, genes expressed in female tissues such as ovary and placenta were located on the $\mathrm{X}$ chromosome more frequently than expected, whereas genes expressed in testes showed the opposite trend. On the surface, this result supports feminization of the X. Complicating this interpretation, however, a previous study by Wang et al. ${ }^{3}$ reported the opposite result: genes expressed specifically in male germ cells were X-linked much more frequently than expected, supporting Rice's hypothesis.

What could account for these conflicting observations? The key issue, it seems, is timing. Wang et al. focused on male germ cells in the earliest stage of spermatogenesis, before meiosis, when the cells are still diploid and mitotic. By contrast, Khil et al. examined intact testes, of which mitotic germ cells compose only a small percentage. Most germ cells in the testes are in various stages of meiosis and are subject to a process known as meiotic sex chromosome inactivation (MSCI), in which the sex chromosomes become heterochromatic and transcriptionally inactive $e^{4}$. The reason MSCI occurs is unknown, but it avoids problems that might occur if nonequivalent sex chromosomes tried to recombine using autosomal, sequence-dependent pairing mechanisms ${ }^{5}$. Because of MSCI, genes expressed during meiosis cannot reside on the $\mathrm{X}$ or $\mathrm{Y}$ chromosomes because they would be inactivated by this chromosomewide silencing mechanism.

To examine whether MSCI accounts for the observed paucity of testes-expressed genes on the X chromosome, Khil et al. used Spo11 knockout mice, in which spermatogenesis is blocked early in meiosis I, at the onset of MSCI. Using microarrays, they compared expression profiles in testes of Spo11 $1^{-/-}$and wild-type mice. They found that genes expressed before MSCI are over-represented on the X chromosome and genes expressed after MSCI are under-represented. In the testes, then, timing is indeed everything: genes expressed early during spermatogenesis are enriched on the X chromosome, in accordance with Rice's hypothesis, and genes expressed later are prevented from residing on the X chromosome by MSCI (Fig. 1).

\section{Conflicts and resolutions}

The Spo11 data set suggests that the $\mathrm{X}$ is masculinized with respect to testes-enriched genes not expressed during spermatogenic meiosis.

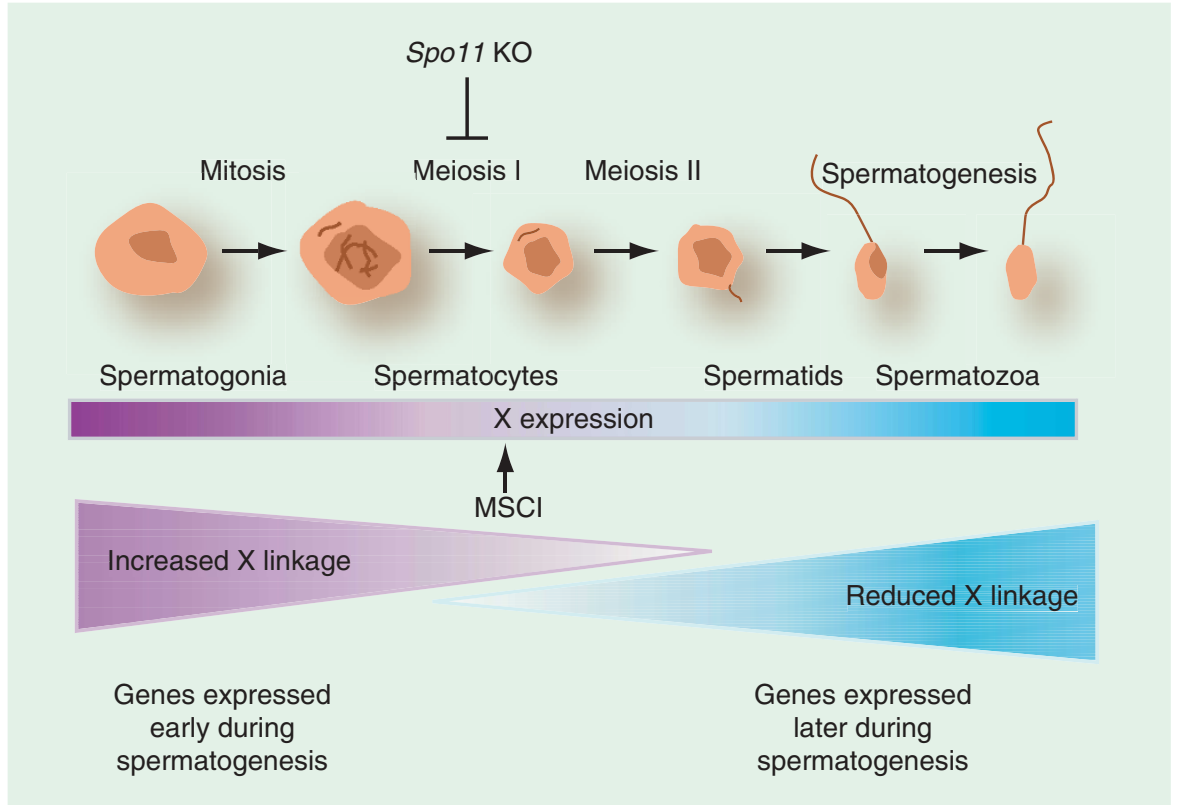

Figure 1 Genes expressed during early spermatogenesis (before $\mathrm{MSCl}$ ) are enriched on the $\mathrm{X}$ chromosome (purple), in accordance with Rice's hypothesis. The onset of $\mathrm{MSCl}$ during early meiosis prevents genes expressed in male germ cells after this time from residing on the $\mathrm{X}$ chromosome (blue).

But this masculinization of the $\mathrm{X}$ chromosome for some genes can occur simultaneously with feminization of the $\mathrm{X}$ chromosome for others, as increased $\mathrm{X}$-chromosome localization is also seen for genes with preferential expression in two female-specific tissues, ovary and placenta $^{2}$. Both of these tissues are composed primarily of somatic cells, with germ cells comprising only a small fraction of the total mass of the ovary. In all somatic tissues, dosage compensation occurs to equalize gene expression between the sexes. For unknown reasons, however, dosage compensation is not implemented in germ cells, and so the sex chromosomes have nonequivalent expression levels between males and females. Once MSCI occurs in male germ cells, the imbalance in sex-linked gene expression is further exacerbated. This imbalance probably results in sensitivity of the sex chromosomes to distinct selective pressures in the germ line relative to the soma. Thus, it is possible that one force (feminization) predominates in the soma and another (masculinization) predominates in the germ line.

Global analysis of sex-biased expression has also been done for Caenorhabditis elegans and Drosophila melanogaster ${ }^{6,7}$, and in both species, demasculinization of the $\mathrm{X}$ chromosome has occurred. These observations, together with the findings of Khil et al., suggest that expression from the $\mathrm{X}$ chromosome in each organism is subject to a curious mosaic of influences. Some superficial parallels exist between the species, and the more divergent organisms seem to be more similar in sex chromosome composition. In both C. elegans and mice, demasculinization of the $\mathrm{X}$ chromosome seems to be primarily driven by $\mathrm{MSCI}^{2,8}$. In flies, however, MSCI does not have a major role; instead, a gene-by-gene selective mechanism acts in both the soma and germ line to demasculinize the $\mathrm{X}$ chromosome ${ }^{7}$. Consistent with this observation, MSCI-dependent imprinting of the $\mathrm{X}$ chromosome occurs in paternal germ cells of worms and mice, but apparently not in flies ${ }^{9,10}$.

Together, the data from global expression studies $^{2,6,7}$ indicate that the $\mathrm{X}$ chromosome is buffeted by prevailing selective forces that vary from one organism to the next. Ultimately, the forces governing gene composition of the sex chromosomes probably reflect divergent mechanisms controlling reproduction, dosage compensation and sex determination in each organism.

1. Rice, W.R. Evolution 38, 735-742 (1984).

2. Khil, P.P., Smirnova, N.A., Romanienko, P.J. \& Camerini-Otero, R.D. Nat. Genet. 36, 642-646 (2004).

3. Wang, P.J., McCarrey, J.R., Yang, F. \& Page, D.C. Nat. Genet. 27, 422-426 (2001).

4. Lifschytz, E. \& Lindsley, D.L. Proc. Natl. Acad. Sci. USA 69, 182-186 (1972).

5. McKee, B.D. \& Handel, M.A. Chromosoma 102 71-80 (1993).

6. Reinke V., San Gil, I., Ward, S. \& Kazmer, K. Development 131, 311-323 (2004).

7. Parisi, M. et al. Science 299, 697-700 (2003).

8. Kelly, W.G. et al. Development 129, 479-492 (2002).

9. Bean, C.J., Schaner, C.E. \& Kelly, W.G. Nat. Genet. 36, 100-105 (2004).

10. Huynh, K.D. \& Lee, J.T. Nature 426, 857-862 (2003). 